\title{
The Rheumatoid Forefoot: A Mini Review
}

\author{
Eildar Abyar*, Haley McKissack and Michael Johnson \\ Department of Orthopedics, The University of Alabama at Birmingham, USA
}

Submission: September 09, 2018; Published: September 20, 2018

*Corresponding author: Eildar Abyar \& Michael Johnson, Department of Orthopedics, The University of Alabama at Birmingham, 1313 13 ${ }^{\text {th }}$ Street South, HGLD 1201, Birmingham, AL, USA 35205, Tel: 205-930-8339; Fax: 205-930-8344, Email: eabyar@uab.edu mdjohnso@uab.edu

\begin{abstract}
Forefoot deformities are common among patients with rheumatoid arthritis and are often debilitating. Chronic inflammation results in pannus formation, ligamentous weakening, and joint destruction, ultimately resulting in multiple joint deformities and altered biomechanics of the foot. Claw toes and hallux valgus are most common and necessitate operative management if conservative treatment fails. This review discusses the pathophysiology, biomechanical impacts, and treatment options available for rheumatoid deformities of the forefoot. It is imperative that physicians, particularly those who specialize in treatment of foot and ankle pathologies, are well-versed in these concepts to optimize patient outcomes.
\end{abstract}

Keywords: Rheumatoid arthritis; Forefoot; Hallux valgus; Claw toe; Deformity

\section{Introduction}

Rheumatoid arthritis is a chronic systemic inflammatory disease which affects approximately $0.8 \%$ of the population [1]. The forefoot in particular is commonly affected, and an estimated $65 \%$ of patients with rheumatoid arthritis have MTP (metatarsophalangeal) joint involvement within the first three years of disease onset [2]. Classically, patients with rheumatoid arthritis have hallux valgus, toe clawing, medial arch lowering, and broadening of the forefoot, a constellation of symptoms known as pied douloureux des rhumatisants [3]. The forefoot plays an integral role in weight-bearing and gait, and therefore understanding the potential consequences of deformities secondary to rheumatoid arthritis as well as treatment options is imperative for optimal management. A variety of treatment options are available, including conservative and surgical options. When conservative treatment fails, operative management must be considered, which may include metatarsal head resection, MTP resection arthroplasty, metatarsal or phalanx shortening, or PIP joint arthrodesis for claw toe deformity [1,2]. First MTP joint arthrodesis or prosthetic implantation may be considered for treatment of hallux valgus [2]. This review aims to describe the pathophysiology of the most common forefoot deformities in patients with rheumatoid arthritis, as well as available treatment options.

\section{Discussion}

\section{Pathophysiology}

In rheumatoid arthritis, chronic autoimmune inflammation leads to joint destruction and deformities $[1,2]$. The autoimmune response targets the synovial membrane, resulting in synovial inflammation and pannus formation. Over time, the pannus erodes the underlying articular cartilage and bone [1]. As the disease progresses, joint capsules, ligaments, and ligamentous insertions are also affected, and the integrity of joints is compromised. In the foot specifically, common secondary deformities include peritalar subluxation and hindfoot malalignment [1], hallux valgus [1,2], Varus deformity of the fifth MTP joint [2,3], and MTP joint dislocation [2].

Toe clawing and hallux valgus are the two most common joint deformities of the forefoot in rheumatoid arthritis [4]. Joint laxity leads to repetitive dorsal MTP joint dislocation with walking [1]. With chronic dorsal dislocation of the proximal phalanges, an imbalance of forces exerted by the intrinsic and extrinsic muscles occurs [1-3] along with extension contractures [3], and permanent dorsiflexion at the MTP joints gradually ensues. Plantarflexion of the distal phalanges occurs simultaneously, also secondary to the imbalance between forces of the intrinsic and extrinsic muscles of the foot [1,2]. This results in clawing, which diminishes the support that the lesser metatarsals provide to the first ray. Lack of lateral support leads to gradual medial deviation of the first metatarsal and resultant hallux valgus [1].

As its anatomical structure is altered, the foot becomes subject to biomechanical changes. With hyperextension of the MTP joints, the plantar plate is stretched, and the metatarsal heads protrude. In weight-bearing in the native foot, the plantar fat pad plays an important role in minimizing stresses transmitted to the metatarsal heads, as well as in reducing pressure on the inferior subcutaneous tissue and plantar skin. However, when the fat pad shifts, stresses transmitted through the metatarsal heads increase, leading to metatarsalgia and 
difficulty walking. Additionally, in the absence of the fat pad as an intermediate cushion, the metatarsal heads exert increased pressure on inferior subcutaneous tissues which may cause callouses and painful bursae to form [1,2]. Redistribution of forces in weight-bearing also occurs with development of hallux valgus. As the intermetatarsal angle between the first and second ray increases, the normal weight-bearing capacity of the first ray is lost, and the second metatarsal is subjected to increased loading. This may result in synovitis and eventual fracture, subluxation, deformity, or dislocation of the second metatarsal [2].

\section{Management}

Non-operative management may first be initiated for symptomatic relief and to prevent development or progression of foot deformities. A variety of pharmacologic agents are available, including disease modifying anti-rheumatic drugs (DMARDs), [1] which slow the clinical progression of rheumatoid arthritis and associated joint damage $[1,4]$. Intra-articular corticosteroid injections are also a viable non-operative option, and have been shown to provide symptomatic pain relief and improve gait in some studies. However, the risks of injections must also be understood, as they may worsen instability in the forefoot. Physical therapy, insoles, shoe inserts, and rocker bottom shoes are potential interventions which may be beneficial. However, conflicting evidence exists as to whether they are effective [1]

When non-operative management is unsuccessful, surgery should be considered. The goal of operative treatment is to relieve pain and correct the deformity while restoring function [2]. Another objective is to restore normal pressure distribution by repositioning the plantar plate and fat pad beneath the metatarsals. The gold standard operative treatment for deformities involving the lesser metatarsals is resection of the metatarsal heads [1,2]. Generally, to prevent metatarsalgia, all four lesser metatarsal heads are resected rather than only those that are symptomatic [2]. Surgery is frequently indicated for all five metatarsals due to the high incidence of hallux valgus, in which forefoot reconstruction has been shown to be effectively performed with first MTP fusion, lesser metatarsal head resection, and PIP joint fusion [5].

Although metatarsal head resection is the most common treatment option and gold standard for deformities of the lesser rays [1,2], the metatarsal heads are important structures for weight-bearing. Thus, techniques have been developed to preserve the heads during soft tissue repositioning, including MTP resection arthroplasty, tendon releases, shortening of the phalanges and metatarsals, and shortening arthrodesis of the PIP joints. The Stains by procedure, which entails excision of the proximal half of the proximal phalanx as well as extensor tenotomies, preserves the metatarsal heads while restoring native position of the plantar fat pad. A case series by Bass et al. demonstrated improved pain scores and functionality among 12 patients who underwent first TMT arthrodesis in combination with the Stains by procedure [4]. Although larger prospective studies are warranted, these results may indicate a potentially safe and effective approach for preserving the metatarsal heads.

Treatment of hallux valgus deformity may be accomplished through resection arthroplasty, first MTP fusion, or prosthesis implantation in the first MTP joint. MTP joint fusion has been proposed to be advantageous in that it protects the lesser MTP joints from excessive dorsiflexion and reduces load-bearing on the lesser metatarsals. However, it is a technically challenging procedure with up to $30 \%$ reported rate of nonunion. Silicone prosthetics have also been used as they preserve joint motion and have demonstrated significant reduction in pain levels. Nonetheless, overall satisfaction rates have been inconsistent, and an associated risk of particulate synovitis exists [2]. Because the first ray plays an integral role in weight-bearing, it is important to choose the appropriate procedure to optimize postoperative biomechanics of the foot.

Complications that may occur with operative treatment of rheumatic forefoot deformities should always be considered so that the risks and benefits of surgery may be compared. Vasculitis weakened soft tissue, and chronic use of immunosuppressants predispose these patients to impaired wound healing and infection. Yano et al. [6] reported delayed wound healing in $20.8 \%$ of a cohort of 192 patients with rheumatoid arthritis, and found significant associations with operative time, number of toes operated on, and number of surgical wounds. Unfortunately, patients with rheumatoid arthritis often have deformities of multiple (if not all) toes, warranting multiple incisions and necessitating longer operative time [6]. Vasculitis can also lead to vascular compromise and subsequent gangrene. Osteopenia is common among patients with rheumatoid arthritis, which can contribute to malunion. Additionally, although surgery can correct articular deformities, it cannot treat the underlying disease and deformities are therefore likely to recur [1].

\section{Conclusion}

Joint deformities of the forefoot caused by rheumatoid arthritis are debilitating for patients and require meticulous attention and care by the physician. Due to the high frequency of these deformities within this population, foot and ankle surgeons should be aware of treatment options, risks, and benefits. The medical community should continue to seek treatment options and procedures to optimize outcomes for patients with forefoot deformities secondary to rheumatoid arthritis.

\section{Conflict of Interest}

The authors have no conflicts of interest to disclose.

\section{References}

1. Jeng C, Campbell J (2008) Current Concepts Review: The Rheumatoid Forefoot. Foot Ankle Int 29(9): 959-968.

2. Louwerens JWK, Schrier JCM (2013) Rheumatoid forefoot deformity: Pathophysiology, evaluation and operative treatment options. Int Orthop 37(9): 1719-1729. 
3. Dave MH, Mason LW, Hariharan K (2015) Forefoot Deformity in Rheumatoid Arthritis: A Comparison of Shod and Unshod Populations. Foot Ankle Spec 8(5): 378-383.

4. Bass EJ, Shariff R, Sirikonda SP (2014) Rheumatoid forefoot reconstruction: Outcome of 1st metatarsophalangeal joint fusion and the Stainsby procedure in the lesser toes. Foot 24(2): 56-61.
5. Whitt KJ, Rincker SA, Hyer CF (2016) Sustainability of Forefoot Reconstruction for the Rheumatoid Foot. J Foot Ankle Surg 55(3): 583585 .

6. Yano K, Ikari K, Takatsuki Y, Taniguchi A, Yamanaka H, et al. (2016) Longer operative time is the risk for delayed wound healing after forefoot surgery in patients with rheumatoid arthritis. Mod Rheumatol 26(2): 211-215

Your next submission with Juniper Publishers
will reach you the below assets
- Quality Editorial service
- Swift Peer Review
- Reprints availability
- E-prints Service
- Manuscript Podcast for convenient understanding
- Global attainment for your research
- Manuscript accessibility in different formats
( Pdf, E-pub, Full Text, Audio)
- Unceasing customer service
Track the below URL for one-step submission
https://juniperpublishers.com/online-submission.php

